\title{
NUMERICAL RELATIVITY: SOLVING GEODESICS EQUATION FOR MASSIVE PARTICLE AROUND BLACK HOLES HORIZON
}

\author{
Shohel Ahmed ${ }^{1}$ and Md. Showkat $\mathrm{Ali}^{2}$ \\ ${ }^{1}$ Department of Mathematics, Bangladesh University of Engineering \& Technology, Dhaka-1000 \\ ${ }^{2}$ Department of Mathematics, University of Dhaka, Dhaka-1000, Bangladesh \\ Corresponding author: Shohel08du@gmail.com
}

Received 21.12.2014

Accepted 28.03.2015

\begin{abstract}
General relativity is the most beautiful physical theory ever invented. It describes one of the most pervasive features of the world we experience - gravitation. The gravitational field acts on nearby matter defines by the curvature of space-time. The black holes of nature are the most perfect macroscopic objects there are in the universe that constructed our concept of space-time. In this paper we use Einstein's general relativity to model the motions of massive particles around the two black holes: static and rotating. These equations of motion around black holes will be studied with special focus towards the variation of symmetry by the change of gravitational effect.
\end{abstract}

Keywords: Static and rotating black holes, Geodesics equation, Schwarzschild solution, Kerr solution

\section{Introduction}

Black holes are a class of gravitational objects predicted by Einstein's theory of general relativity given by him in 1915. And since then general theory of relativity provides only a single unique family of solutions for their descriptions. In $1916 \mathrm{~K}$. Schwarzschild gave the first solution for the Einstein equation of general relativity. His solution described the space-time around a static massive black hole and was called the Schwarzschild-metric. Later, in 1963, R. Kerr discovered another solution: the Kerr-metric. The Kerr-metric gives the space-time outside a massive rotating black hole. The solution of the field equations has a mathematical sophistication: the so-called metric that describe curved space-time around non-spinning and spinning centers of gravitational attraction. The every possible question about trajectories of massive particles around a gravitational field is described by the geodesic equation

$$
\frac{d^{2} x^{\mu}}{d \tau^{2}}+\Gamma_{v \sigma}^{\mu} \frac{d x^{v}}{d \tau} \frac{d x^{\sigma}}{d \tau}=0
$$

Where $\tau$ is the proper time along the trajectory measured from some point on it. $x^{\mu}(\mu=0,1,2,3)$ is a general coordinate system and one of these is a time-like coordinate. 
A tremendous amount of algebra is involved in doing calculation in general relativity. It is almost a requirement that some computer algebra program be used to do the calculations. Some Mathematica codes had been developed by Baumann [2], Hartle [3] and Zimmerman [6]. Throughout this paper all of the orbits produced will be shown visually by Mathematica 9.

\section{Schwarzschild Solution}

One of the simplest solutions to the Einstein equations corresponds to a metric that describes the empty space-time outside a static spherical body of mass $\mathrm{m}$ is Schwarzschild solution. Which interpreted as the field of a highly collapsed spherically symmetric black hole [1]. The metric is given in the form:

$$
d s^{2}=-\left(1-\frac{2 m}{r}\right) d t^{2}+\left(1-\frac{2 m}{r}\right)^{-1} d r^{2}+r^{2} d \theta^{2}+r^{2} \operatorname{Sin}^{2} \theta d \varphi^{2}
$$

In metric (2) Schwarzschild radius $r_{s}=2 m$ is called an event horizon or just a horizon [4] which acts like a "one-way membrane". The overall technique here is to compute the geodesic equation from the Christoffel symbols (with Mathematica doing all the algebraic legwork). From this, differential equations will be derived that can be solved with the appropriate initial conditions $\frac{d x^{\mu}}{d \tau}$. Different techniques are mentioned to solve this system of $2^{\text {nd }}$ order differential equations, Runge-Kutta mentioned in Thomas [8], Shooting method in Plebanski [5]. Using the Christoffel symbols we can compute geodesic equations for Schwarzschild solution:

$\frac{d^{2} t}{d \tau^{2}}=\frac{2 m r^{\prime} t^{\prime}}{2 m r-r^{2}}$

$\frac{d^{2} r}{d \tau^{2}}=\frac{\left.-m r^{2}\left(r^{\prime}\right)^{2}+(-2 m+r)^{2}\left(m\left(t^{\prime}\right)^{2}-r^{3}\left(\theta^{\prime}\right)^{2}+\sin [\theta]^{2}+\left(\phi^{\prime}\right)^{2}\right)\right)}{(2 m r-r) r^{3}}$

$\frac{d^{2} \theta}{d \tau^{2}}=-\frac{2 r^{\prime} \theta^{\prime}}{r}+\cos [\theta] \sin [\theta]\left(\phi^{\prime}\right)^{2}$

$\frac{d^{2} \Phi}{d \tau^{2}}=-\frac{2\left(r^{\prime}+r \cot [\theta] \theta^{\prime}\right) \phi^{\prime}}{r}$

Now we supply initial conditions for geodesics equations and solve for the coordinates parameterized in $\tau$. We provide initial displacement $x^{\mu}(0)=\{0,6.5, \pi / 2,0\}$ and initial velocities in all three spatial coordinates $\frac{d x^{\mu}(0)}{d \tau}=\{0,0,0.088\}$. By using the general tensor form $g_{\mu \nu} u^{\mu} u^{\nu}=-1$ for massive particles we compute the initial condition for $\frac{d t}{d \tau}$ [7]. Mathematica cannot generate a general analytic solution, so we resort to numerical solution using NDSolve by taking $m=1$. We have the following plots using proper time $\tau$ up to 2000: 

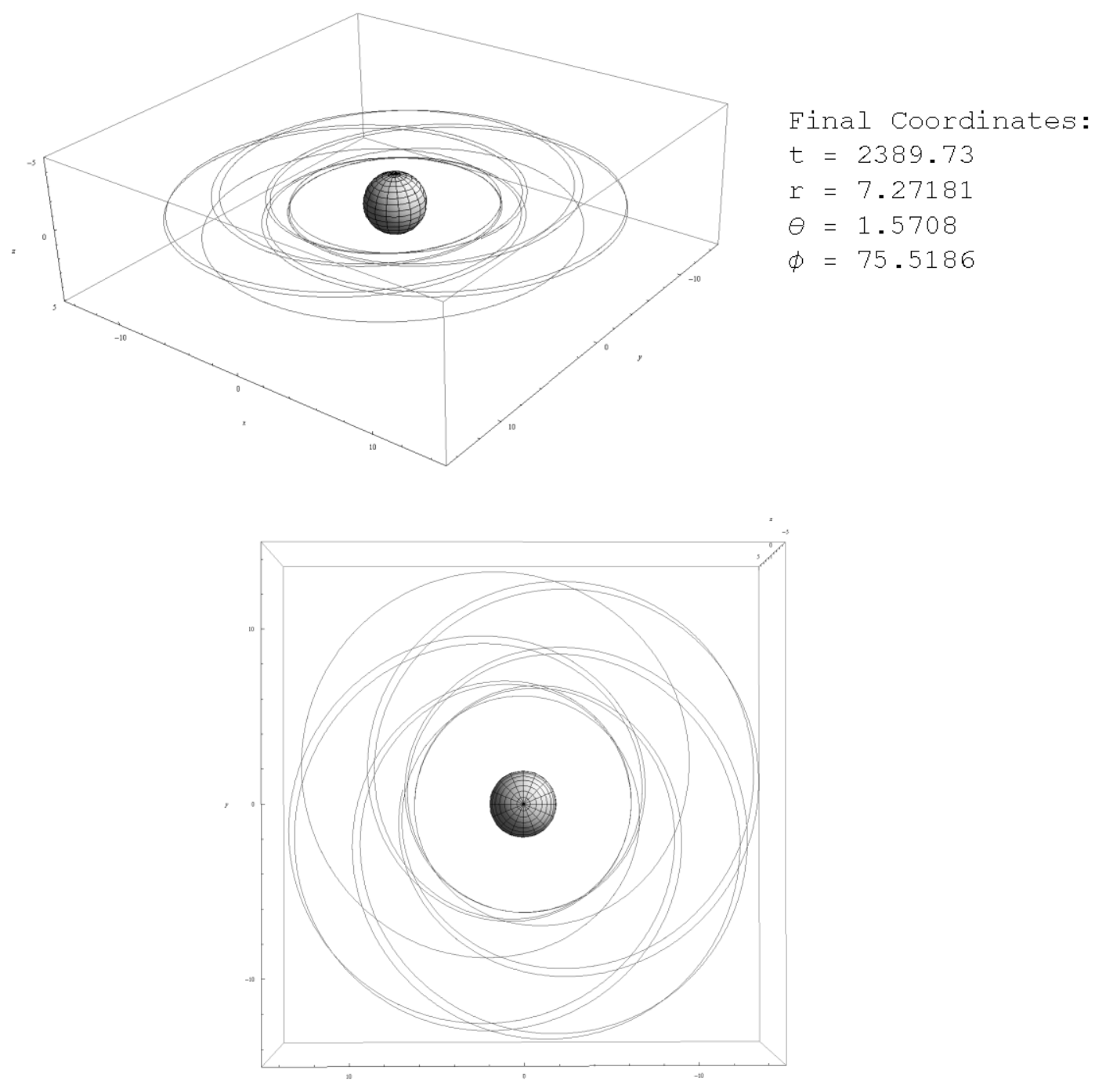

In the above plots a different set of initial conditions help us to understand what is occurring at the horizon. The sphere has a radius equal to the horizon $r=2 \mathrm{~m}$. In Schwarzschild coordinates there is a singularity at the horizon and hence, numerical equation solving cannot cross the horizon. We see clear evidence of precession, as expected in the Schwarzschild metric close to the horizon. Also the modest time dilation for this orbit, while the proper time $\tau$ goes to 2000, the coordinate time $t$ extends up to 2400. This is a sign that the equations are properly solved and numerical errors are not significantly disrupting the orbit. One final point is to find out the inherent spherical symmetry in the Schwarzschild geometry. The following plot is made with initial velocities in all three spatial coordinates 
$\frac{d x^{\mu}(0)}{d \tau}=\{-0.8,0.035,0.08359\}$ and initial displacement $x^{\mu}(0)=\{0,10, \pi / 4,0.2\}$, while the proper time $\tau$ goes to 5000:
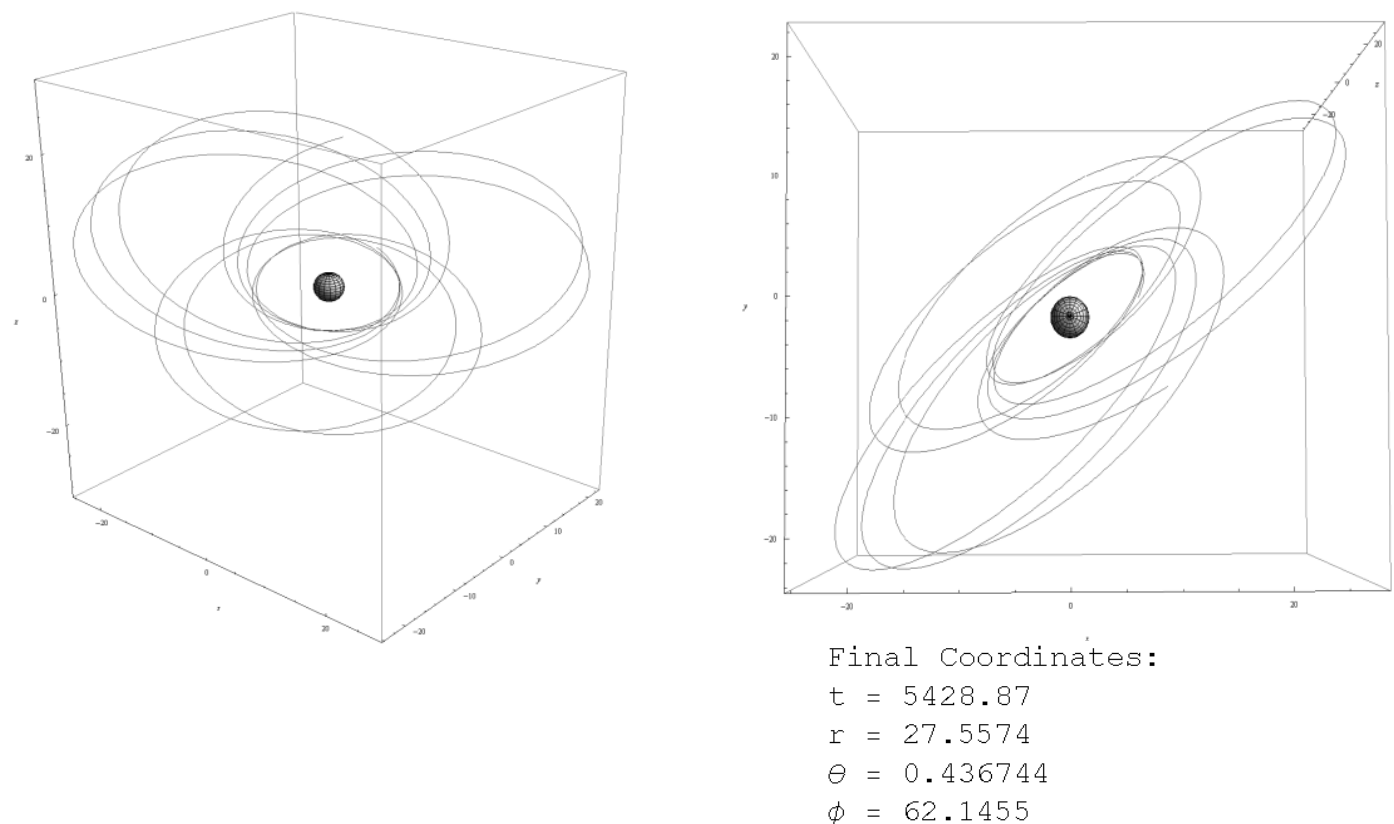

It is clear from the above plot that the orbit is still in a plane, although it is now tipped with respect to the z-axis. This is not expected in the general Kerr geometry that has a preferred rotational axis.

\section{Kerr Solution}

Stars that have exhausted their thermonuclear fuel are no longer able to maintain their equilibrium with their inward gravitational force. The star will undergo a gravitational collapse. If the star is massive enough, its final state will be as a black hole. And because the star rotates before the collapse, the collapse will result in a spinning or rotating black hole. The space-time around the final state of a very massive star is described by the Kerr-metric [4]. The Kerr metric using BoyerLindquist coordinates given in the form:

$$
\begin{aligned}
& d s^{2}=-\left(1-\frac{2 m r}{\sum_{1}}\right) d t^{2}+\frac{4 a m r \sin ^{2} \theta}{\sum_{1}} d \varphi d t+\left(\frac{2 m a^{2} r \sin ^{2} \theta}{\sum_{1}}+r^{2}+a^{2}\right) \sin ^{2} \theta d \varphi^{2}+\sum_{1}\left(\frac{d r^{2}}{\sum_{2}}+d \theta^{2}\right) \\
& \text { where } \quad \sum_{1} \equiv\left(r^{2}+a^{2} \cos ^{2} \theta\right) \\
& \quad \sum_{2} \equiv\left(r^{2}-2 m r+a^{2}\right)
\end{aligned}
$$

This section is about the paths of free test particles outside a Kerr black hole. As these are freely falling particles, they are described by geodesics. 
$\frac{d^{2} t}{d \tau^{2}}=\left(4 m\left(2 a^{2} r\left(a^{2}+r(-2 m+r)\right) \sin [2 \theta] \theta^{\prime}\left(t^{\prime}-a \sin [\theta]^{2} \phi^{\prime}\right)+r^{\prime}\left(\left(a^{2}+r^{2}\right)\left(a^{2}-2 r^{2}+a^{2} \cos [2 \theta]\right) t^{\prime}\right.\right.\right.$

$\left.\left.\left.+a\left(-a^{4}+3 a^{2} r^{2}+6 r^{4}+\left(-a^{4}+a^{2} r^{2}\right) \cos [2 \theta]\right) \sin [\theta]^{2} \phi^{\prime}\right)\right)\right) /\left(a^{2}+r(-2 m+r)\right)\left(a^{2}+2 r^{2}+a^{2} \cos [2 \theta]\right)^{2}$

$\frac{d^{2} r}{d \tau^{2}}=\left(-\frac{\left(r^{2}+a^{2} \cos [\theta]^{2}\right)^{2}\left(r\left(a^{2}-m r\right)+a^{2}(m-r) \cos [\theta]^{2}\right)\left(r^{\prime}\right)^{2}}{a^{2}+r(-2 m+r)}+m\left(a^{2}+r(-2 m+r)\right)\right.$

$\left(-r^{2}+a^{2} \cos [\theta]^{2}\right)\left(t^{\prime}\right)^{2}+2 a^{2} \cos [\theta]\left(r^{2}+a^{2} \cos [\theta]^{2}\right) \sin [\theta] r^{\prime} \theta^{\prime}+r\left(a^{2}+r(-2 m+r)\right)\left(r^{2}+a^{2} \cos [\theta]^{2}\right)^{2}$

$\left(\theta^{\prime}\right)^{2}-2 a m\left(a^{2}+r(-2 m+r)\right)\left(-r^{2}+a^{2} \cos [\theta]^{2}\right) \sin [\theta]^{2} t^{\prime} \phi^{\prime}+\left(a^{2}+r(-2 m+r)\right) \sin [\theta]^{2}\left(r^{5}+a^{4} r \cos [\theta]^{4}\right.$

$\left.\left.-a^{2} m r^{2} \sin [\theta]^{2}+\cos [\theta]^{2}\left(2 a^{2} r^{3}+a^{4} m \sin [\theta]^{2}\right)\right)\left(\phi^{\prime}\right)^{2}\right) /\left(r^{2}+a^{2} \cos [\theta]^{2}\right)^{3}$

$\frac{d^{2} \theta}{d \tau^{2}}=\left(-\frac{a^{2} \cos [\theta]\left(r^{2}+a^{2} \cos [\theta]^{2}\right)^{2} \sin [\theta]\left(r^{\prime}\right)^{2}}{a^{2}+r(-2 m+r)}+a^{2} m \sin [2 \theta]\left(t^{\prime}\right)^{2}-2 r\left(r^{2}+a^{2} \cos [\theta]^{2}\right)^{2} r^{\prime} \theta^{\prime}\right.$

$+a^{2} \cos [\theta]\left(r^{2}+a^{2} \cos [\theta]^{2}\right)^{2} \sin [\theta]\left(\theta^{\prime}\right)^{2}-2 a m r\left(a^{2}+r^{2}\right) \sin [2 \theta] t^{\prime} \phi^{\prime}-\cos [\theta]\left(r^{2}+a^{2} \cos [\theta]^{2}\right)^{2} \sin [\theta]$

$\left.\left(-a^{2}-r^{2}-\frac{4 a^{2} m r \sin [\theta]^{2}}{r^{2}+a^{2} \cos [\theta]^{2}}-\frac{2 a^{4} m r \sin [\theta]^{4}}{\left(r^{2}+a^{2} \cos [\theta]^{2}\right)^{2}}\right)\left(\phi^{\prime}\right)^{2}\right) /\left(r^{2}+a^{2} \cos [\theta]^{2}\right)^{3}$

$\frac{d^{2} \phi}{d \tau^{2}}=\left(-\left(a^{2}+r(-2 m+r)\right) \cot [\theta] \theta^{\prime}\left(-16 a m r t^{\prime}+\left(3 a^{4}+8 a^{2} m r+8 a^{2} r^{2}+8 r^{4}+4 a^{2}\left(a^{2}+2 r(-m+r)\right)\right.\right.\right.$

$\left.\left.\cos [2 \theta]+a^{4} \cos [4 \theta]\right) \phi^{\prime}\right)+2 r^{\prime}\left(4 a m\left(r^{2}-a^{2} \cos [\theta]^{2}\right) t^{\prime}+\left(-8 m r^{4}+4 r^{5}-8 a^{2}(m-r) r^{2} \cos [\theta]^{2}+\right.\right.$

$\left.\left.\left.\left.4 a^{4} r \cos [\theta]^{4}-4 a^{2} m r^{2} \sin [\theta]^{2}+a^{4} m \sin [2 \theta]^{2}\right) \phi^{\prime}\right)\right) / a^{2}+r(-2 m+r)\right)\left(a^{2}+2 r^{2}+a^{2} \cos [2 \theta]\right)^{2}$

We will look at more complicated orbits in three dimensions for a Kerr black hole near the $\frac{a}{m}=1$ limit. The sphere and red circles here no longer correspond to the Schwarzschild radius, but instead the outer horizon $\sum_{+}=m+\sqrt{m^{2}-a^{2}}$ from Hartle [3]. Providing same initial displacement and initial velocities as in section- 2 we have the following plot:

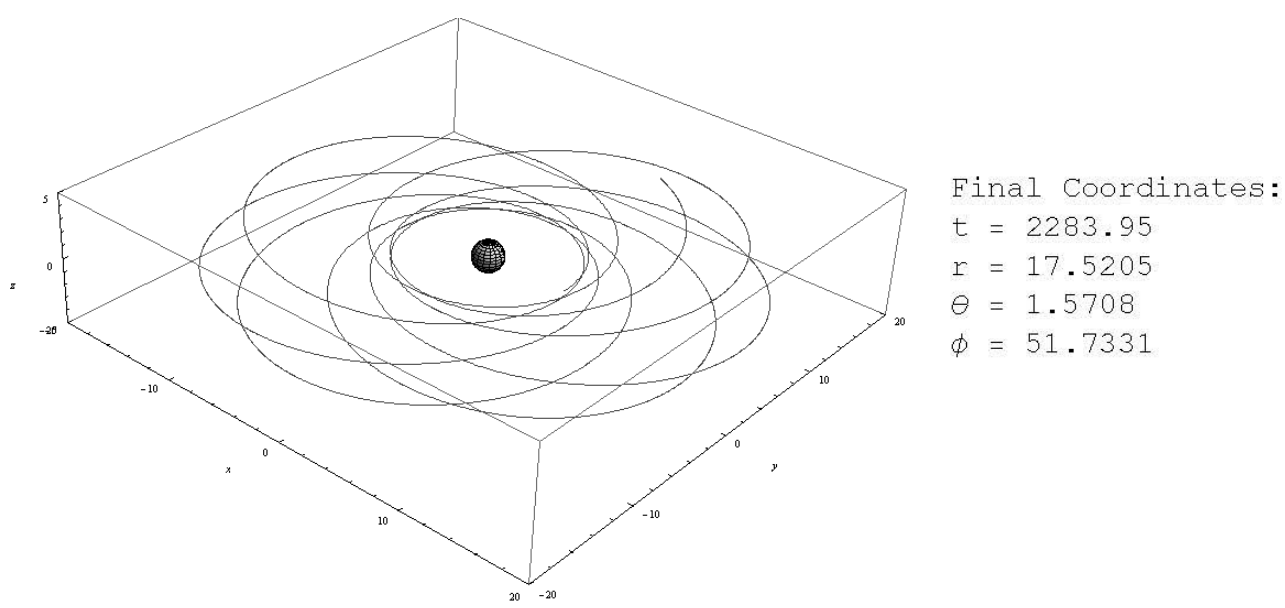


Now in the following plot we examine the case for $a=0$ i.e. with no angular momentum Kerr solution reduces to Schwarzschild solution (same as section-2).
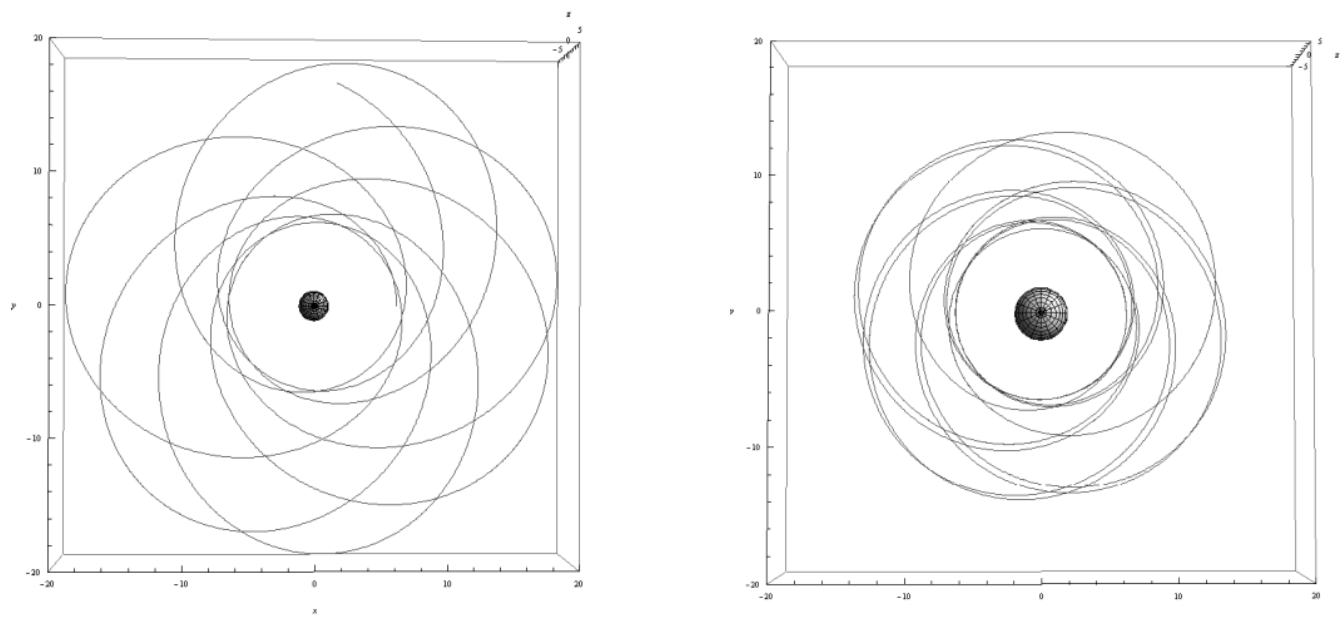

Now we set initial conditions to provide velocities in all three coordinates so as to allow for a more complicated orbit. The initial conditions taken same as in section-2 for compares the cases in the following plot:
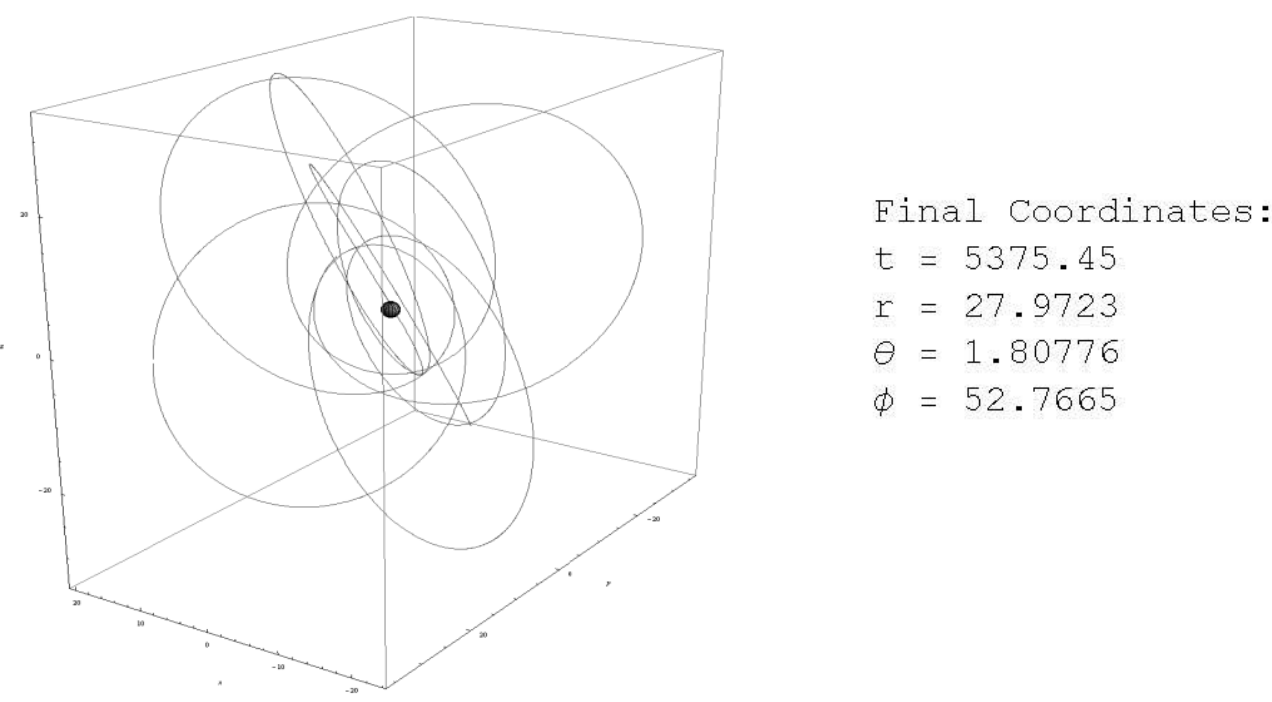

Here we finally see orbits completely like anything at all familiar. By orbiting in a plane other than the equatorial plane, the rotational effects of the black hole pull the orbit into a shape that has no obvious symmetry properties, unlike the Schwarzschild case, where the coordinate system could always be re-oriented to lie in a plane. Despite these oddities, the time dilation is not too extreme $(=1.08)$, so this orbit is indeed the free-fall orbit in the Kerr geometry. Again in the following plot we compare the case when $a=0$ in Kerr solution which leads us to Schwarzschild solution: 

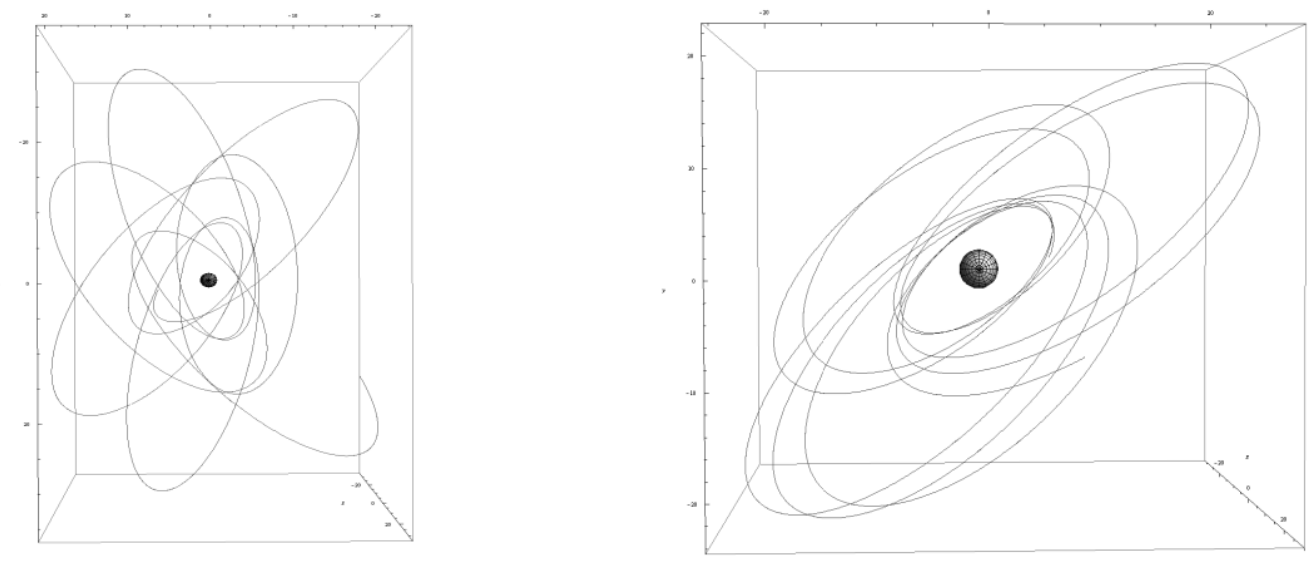

\section{Conclusion}

General Relativity flows from Einstein's field equations, which relate the mass and pressure in a region of space-time to the "warping" of space-time across the region of a black hole. The field equations reveal how this warping is experienced by any particle, whether the black hole is moving, accelerating, rotating, stretching, twisting, or tumbling. We have examined a wide variety of particle orbits both in the Kerr and Schwarzschild geometry. The Kerr geometry is described by a far more complicated metric than the Schwarzschild geometry to the Einstein's equations. The initial experimental goal was achieved, that is highly asymmetric orbits arises from Kerr geometry, which normally have relatively simple paths in Schwarzschild geometry. A fascinating follow-on to this paper would be that of determining changes of rotational effect from the Kerr geometry to Schwarzschild geometry. That is, the Kerr solution with zero angular momentum tends to the Schwarzschild solution representing the exterior field of a spherically symmetric source.

\section{REFERENCES}

[1] Foster, J. \& Nightingale, J.D. A Short Course in General Relativity, third edition, New York, Springer, 2006.

[2] Gred Baumann, Mathematica for Theoretical physics, third edition, Springer Science and Business Media, New York, 233 Springer Street, 2005.

[3] James B Hartle, Gravity an Introduction to Einstein's General Relativity, Addison Wesley, San Francisco, 2003.

[4] Jamal Nazrul Islam, Rotating Fields in General Relativity, Cambridge University Press, 1985.

[5] Jery Plebanski, Andrzey Krasinski, An Introduction to General Relativity and Cosmology, Cambridge University Press, The Edinburgh Building, CB2 2RU, 2006.

[6] Robert L. Zimmerman, Fredrick I. Olness, Mathematica for Physics, second edition, Addison Wesley, San Francisco, 2002.

[7] Shohel Ahmed, The Rotating Fields in General Relativity, M.S. thesis under Department of Mathematics, University of Dhaka in 2014.

[8] Thomas W. Baumgarte \& Stuart L. Shapiro, Numerical Relativity Solving Einstein's Equations on the Computer, Cambridge University Press, The Edinburgh Building, CB2 8RU, 2010. 